\title{
DISREGULACIÓN EMOCIONAL Y EMOCIONES MORALES EN EDUCACIÓN: APORTACIONES DESDE LA NEUROPSICOLOGÍA
}

\author{
Claudia Godínez Castillo* \\ Benemérita Universidad Autónoma de Puebla, México \\ claudiagodica@gmail.com \\ Dulce María Carolina Flores Olvera**. \\ Benemérita Universidad Autónoma de Puebla, México \\ flores4@gmail.com
}

Recibido: 31/10/2018 Aceptado: 4/03/2019

\section{Resumen}

La propuesta educativa de formar sujetos integrales ha permitido la entrada de las emociones a la agenda educativa. Esto también ha permitido la inter y multidisciplina, por lo que la educación se ha nutrido de otras áreas como la neuropsicología. El presente escrito pretende reconocer la relación entre disregulación emocional y emociones morales en el ámbito educativo. Para ello se parte de la comprensión de la emoción como elemento central en procesos cognosocioafectivos, para decantar en las emociones morales, que son de particular interés para la educación, por su intención formativa y su búsqueda de entablar un diálogo entre lo gnosológico, lo axiológico, la reflexión constante y la búsqueda de principios. Finalmente se aborda cómo la disregulación emocional afecta a las emociones morales, provocando decisiones erróneas. Al respecto, la neuropsicología ha otorgado otra mirada al panorama educativo, por lo que mostramos sus aportaciones.

Palabras clave: Disregulación emocional - Emociones morales - Neuroeducación - Procesos cognitivos - Neuropsicología.

\section{Abstract}

The educational proposal in charge of creating complete people has allowed the emotions inside the educational agenda. This has also allowed the inter and multidiscipline, so that education has been enriched by other areas such as neuropsychology. The present script intends to recognize the relation between emotional disregulation an moral emotions within the educational matters. Comprehension of the emotions represents a fundamental in cognosocioafective processes to filter in moral emotions which result of particular interest for education because of its formative attempt and the intention of setting dialoge between knowledge, values, the constant reflection ad the search of principles. Finally this is about how emotional disregulation affects moral emotions resulting in

\footnotetext{
* Licenciada en Psicología por la Universidad Intercontinental Diplomado en Neuropsicología Infantil, y Magíster en Diagnóstico y Rehabilitación Neuropsicológica. Actualmente es Candidata a Doctora en Investigación e Innovación Educativa en la Facultad de Filosofía y Letras, Benemérita Universidad Autónoma de Puebla.

** Doctora en Psicología con especialización en Pedagogía, Universidad Estatal de Moscú. Posdoctorada en la Universidad de Toronto y el CAMH. Profesor-Investigador Titular "A" Tiempo Completo en el Doctorado en Investigación e Innovación Educativa, Facultad de Filosofía y Letras, Benemérita Universidad Autónoma De Puebla.
} 
REVISTA DE LA ESCUELA DE CIENCIAS DE LA EDUCACIÓN, AÑo 15, NRO. 14, VOL. 1, ENERO A JUNIO DE 2019. PÁGINAS 45-52. ISSN 1851-6297 (DESDE DICIEMBRE DE 2006 A DICIEMBRE DE 2017). ISSN 2362-3349 (EN LINEA). DISREGULACIÓN EMOCIONAL Y EMOCIONES MORALES EN EDUCACIÓN: APORTACIONES DESDE LA NEUROPSICOLOGÍA. CLAUDIA GODINEZ · DULCE MARÍA CAROLINA FLORES OLVERA.

wrong decision making, causing particular interest for education. In this matter neuropsychology has shared a different look on the educational field, therefore we present its contributions.

Keywords: Emotional dysregulation - Moral emotions - Neuroeducation - Cognitive processes Neuropsychology.

\section{Emociones en el ámbito educativo}

Es claro que la educación ha dejado de interpretarse como la mera transmisión de datos o del conocimiento; por el contrario, el proceso de enseñanza-aprendizaje se plantea como un proceder permanente. Se concibe como un proceso no acabado en donde todos sus agentes involucrados coconstruyen los saberes culturales-educativos. El proceso educativo lleva implícita la formación profesional en su contenido disciplinar, pero también la formación del sujeto como ser integral.

Por tanto, en el proceso de enseñanza- aprendizaje ya no es suficiente la transmisión de contenido académico o de cuestiones instrumentales. Por el contrario, también se busca formar personas que no sólo sean profesionistas de calidad, sino ciudadanos responsables con conciencia moral desde su ámbito sociocultural, que verdaderamente aporten y contribuyan desde su formación a la construcción de una sociedad más ética y justa, poniendo en evidencia su marco de referencia valoral, más con acciones que con palabras.

El escenario de la educación en este sentido se revela como formador y/o inhibidor del ejercicio socialmente responsable, crítico, reflexivo, moral y ético. La educación siempre mantiene una intención: formativa, investigativa, correctiva; y es necesario buscar una senda propia que permita proponer y encontrar soluciones desde un contexto histórico concreto, teniendo en cuenta la situación global de la humanidad, e identificando la relación que se guarda entre esos contextos particular y general.

A partir de esta mirada conciliadora y de la necesidad de formar individuos integrales, ha tomado relevancia el ámbito socioafectivo. En el ámbito educativo se ha evidenciado la importancia del manejo de las emociones y del afecto para el proceso de enseñanza-aprendizaje. Su demanda ha incorporado teorías y modelos como las inteligencias múltiples, la inteligencia emocional, entre otros. Aun no hay consenso respecto a la conceptualización de las habilidades que de aquí derivan, llamándoseles de manera indiscriminada competencias o habilidades socioafectivas, sociocognitivas socioemocionales, sociales, afectivas, emocionales o cognosocioafectivas.

Al respecto, se sugiere utilizar el término de procesos cognosocioafectivos, derivado de promover y entender como una unidad a las dimensiones cognitivas, sociales y afectivas de un mismo proceso, sin escisiones. Aunado a esto, también resulta pertinente aclarar que dichos procesos habrán de verse traducidos en habilidades cognosocioafectivas. Esto es, el resultado visible de tal proceso es una habilidad expresada en una acción (cargada evidentemente de emoción y pensamiento).

Cabe aclarar que las nombradas ciencias exactas entienden a la emoción como la respuesta fisiológica del organismo expresada en respuestas autonómicas como piloerección, frecuencia cardíaca y respiratoria. Por su parte el afecto o sentimiento se entiende como una respuesta subjetiva atravesada por la cognición. Sin embargo, para los fines de este estudio retomaremos el concepto de emoción y de afecto, más que como sinónimos, como elementos de un todo, puesto que el transitar del aspecto fisiológico al cognitivo se entiende como parte de un mismo proceso, sin pretender caer en dicotomías ni reduccionismos.

Actualmente se comprende que la metodología educativa debe promover ambientes de gozo para la generación óptima del aprendizaje; intervenciones positivas dentro del aula; donde la actitud del docente es fundamental para transmitir pasión y regocijo en el simple hecho de aprender y conocer; o que en el aprendizaje cooperativo existe una opción loable para la gestación de conocimientos académicos y de habilidades cognosocioafectivas.

$\mathrm{Si}$ bien muchas disciplinas han sumado esfuerzos para continuar desarrollando aproximaciones y metodologías ante el fenómeno de las emociones y el afecto, es evidente que se debe seguir trabajando en conjunto hacia estrategias más eficientes. Al respecto, el escenario educativo se ha nutrido de las aportaciones de otras disciplinas. Interesa en este caso la contribución de la neuropsicología, entendida como la rama especializada de la psicología que estudia la relación entre la organización cerebral y los procesos cognoscitivos-comportamentales en su sentido más amplio: acciones, emociones, motivaciones, relaciones sociales, etc.

\section{¿Cómo entiende la Neuropsicología a las emociones?}

En la evolución del estudio de las emociones se ha pasado por la psicobiología, la teoría central de las emociones, la teoría periférica de las emociones de James-Lange, la teoría de las emociones básicas, etc.; instaurando el asiento fisiológico y objetivo de las emociones, así como su capacidad de concientizarlas y el procesamiento emocional en su experiencia y percepción. Gracias a esto, los estudios muestran que la emoción ya no sólo se comprende como los aspectos neurovegetativos, sino también son reconocidos sus componentes conductual y subjetivo-cognitivo.

Actualmente se entiende a la emoción como un fenómeno que refleja las funciones de circuitos que permiten a un organismo sobrevivir y prosperar mediante la detección y la respuesta a los retos más destacados y oportunidades dentro del ambiente (LeDoux, 2012). La emoción para la neuropsicología se define como un proceso complejo que implica ciertos estímulos relevantes como desencadenantes de 
REVISTA DE LA ESCUELA DE CIENCIAS DE LA EDUCACIÓN, AÑo 15, NRO. 14, VOL. 1, ENERO A JUNIO DE 2019. PÁGINAS 45-52. ISSN 1851-6297 (DESDE DICIEMBRE DE 2006 A DICIEMBRE DE 2017). ISSN 2362-3349 (EN LINEA). DISREGULACIÓN EMOCIONAL Y EMOCIONES MORALES EN EDUCACIÓN: APORTACIONES DESDE LA NEUROPSICOLOGÍA. CLAUDIA GODINEZ · DULCE MARÍA CAROLINA FLORES OLVERA.

alguna condición, la existencia de la interpretación subjetiva, procesos valorativos a nivel de cognición, cambios fisiológicos (por ejemplo de activación), y la expresión emocional que moviliza para la acción y tiene como finalidad la adaptación al medio (Fernández-Abascal, Jiménez, Martín, 2003).

Para una mejor comprensión, habrán de entenderse las múltiples funciones de las emociones que propone Mora (2000, p.27) en su heptálogo de las emociones:

1. Las emociones sirven para defendernos de estímulos nocivos o aproximarnos a estímulos placenteros, por lo que son motivadoras.

2. Generan que las respuestas del organismo ante eventos sean polivalentes y flexibles, dotando de mayor versatilidad a la conducta.

3. "Alertan" al individuo como un todo, activando múltiples sistemas cerebrales (activación reticular, atencional, mecanismos sensoriales, motores, procesos mentales), endocrinos (activación suprarrenal medular y cortical y otras hormonas), metabólicos (glucosa y ácidos grasos) y autonómicos (cardiovascular, respiratorio, etc.)

4. Mantienen la curiosidad y la indagación de lo novedoso, ampliando el marco de seguridad para la supervivencia.

5. Sirven como lenguaje básico, creando lazos emocionales con claras consecuencias de éxito para la supervivencia biológica y social.

6. Almacenan y evocan memorias de forma muy eficiente.

7. Juegan un importante rol en el razonamiento, organización y programación de conductas dirigidas a objetivos.

A partir de esto, se plantea y comprende que las emociones dejaron de verse como un sesgo sobre la cognición y la evaluación de los eventos. Por el contrario, ahora se reconoce que las reacciones emocionales permiten coordinar los sistemas de respuesta subjetivos y conductuales; dirigen la conducta y juegan un rol comunicativo al revelar los valores propios y sociales (Martínez, 2008). De acuerdo a Verdejo y Bechara (2009; citado en Chicharro et al, 2011), las emociones también repercuten en el desarrollo del comportamiento y la toma de decisiones adaptativas.

Si bien todas las emociones son importantes, particularmente interesan en este caso las emociones morales, por mantener una relación biunívoca con la educación, ya que se mediatizan y se interpelan cíclicamente. Esto es así porque las emociones morales tienen como eje conductor a la ética, que en su práctica se sustenta en la moral, y cuya aplicación decanta en los valores. Las emociones morales se identifican como preferencias vigentes de la mayoría de los ciudadanos en una sociedad, y están mediadas no sólo por la biología del sujeto, sino sobre todo por la cultura (costumbres, lenguaje, significados, educación, etc.).

\section{Emociones Morales}

Las emociones se han clasificado de acuerdo a su complejidad en emociones orientadas a un objeto, básicas y complejas o sociales (Johnson-Laird y Oatley, 2000; citado en Damasio, 2005). Particularmente respecto a dichas emociones complejas o sociales, Haidt (2003; citado en Mercadillo Díaz, Barrios, 2007, p. 3), propone cuatro familias de emociones morales:

1. Emociones de condena: Involucran la ira, el disgusto, el desprecio y la indignación. Se presentan ante el rompimiento de códigos morales como la ética de la comunidad, la autonomía y la pureza física.

2. Emociones de autoconciencia: Incluyen la vergüenza, el pudor y la culpa. Las dos primeras se presentan ante el reconocimiento del quiebre de una norma social, con una tendencia hacia la reducción de la presencia social, disculpar o justificar la acción. En la culpa, se percibe que el quebrantamiento ha causado sufrimiento a terceros, en un sentido pro-social motiva a revertir los efectos del daño.

3. Emociones relativas al sufrimiento ajeno: Implica a la compasión, surge por la inferencia del sufrimiento o dolor ajeno, y suele accionar conductas altruistas hacia la víctima.

4. Emociones de admiración: Se incluyen la gratitud, la admiración y la devoción, se relacionan con la percepción de algo o alguien considerado bueno, correcto, estético o moralmente ejemplar. La caridad, la lealtad o el auto-sacrificio derivan de estas emociones.

Una característica de gran impacto de las emociones morales es la existencia de la valoración consciente del individuo y la interpretación subjetiva que logra desde la cognición, lo cual permite indagar sobre las motivaciones personales versus el aprendizaje socio-cultural, mucho del cual se gesta en el ámbito educativo. Las emociones morales se desencadenan en respuesta a la percepción subjetiva del quebrantamiento de normas sociales explícitas e implícitas, así como de estereotipos inherentes en los códigos, actitudes y creencias individuales (Haidt, 2003; citado en Mercadillo et al, 2007).

Las emociones morales dan cuenta de una construcción moral social, en donde el sujeto se identifica, construye y deconstruye. A través de estas emociones morales el individuo es capaz de valorar su propio actuar, interpretar y resignificar escenarios sociales y modelos propios de comportamiento asumidos. Dese esta perspectiva, sería prudente revisar la intención formativa -sea consciente o inconsciente- de la educación, sobre todo a sabiendas de que no es ningún secreto que la enseñanza legitimada hasta el 
momento priorice el saber y el saber hacer sobre el saber ser. No obstante nuevos discursos emergen ante las demandas sociales, y buscan entablar un diálogo entre lo gnosológico, lo axiológico, la reflexión constante y la búsqueda de principios desde contextos y sujetos particulares.

Las emociones morales pueden brindar una primera pauta sobre la construcción social moral imperante, y su estudio profundiza sobre la afectación o mantenimiento del comportamiento moral social esperado. Ante el quebrantamiento de tales códigos morales asumidos y construidos socialmente, se pone en evidencia tanto el razonamiento moral individual como la experiencia subjetiva de la emoción.

A esta experiencia subjetiva de la emoción se le ha denominado procesamiento emocional, entendido como el proceso mental que evalúa información emocionalmente relevante, provoca respuestas en el propio cuerpo produciendo un estado corporal emocional, y produce cambios mentales adicionales. En el procesamiento emocional se incluyen habilidades de identificación, comprensión, producción y expresión de emociones, que se relacionan con dimensiones de comportamiento como la percepción selectiva de señales sociales, el cuidado y apego, el reconocimiento de los estados afectivos de los demás, la resolución de problemas en un contexto social y el aprendizaje de las prácticas sociales (Hernández, 2014).

Dentro del procesamiento emocional, se subraya a la regulación emocional como una habilidad cognosocioafectiva de gran impacto en el ámbito educativo. Se considera que, sobre la base del trabajo empírico y conceptual, la regulación emocional debe involucrar la conciencia, comprensión y aceptación de las emociones, la habilidad para controlar conductas impulsivas, comportarse de acuerdo con los objetivos deseados, aun cuando se experimenten emociones negativas, y la habilidad para usar apropiadamente estrategias de regulación emocional flexibles, conforme a la situación y el contexto sociocultural (Gratz y Roemer, 2004).

Por su parte, cuando no se presentan dichos atributos se habla de disregulación emocional, misma que promueve la impulsividad, la compulsión, la generación de deterioro para mantener relaciones sociales, conduce al aislamiento y a juicios morales inapropiados. También altera habilidades representativas para el buen desempeño profesional y académico, como la programación de conductas dirigidas a objetivos, la toma de decisiones adaptativas, o la revaloración emocional, que es una fuerte estrategia de regulación (Van't Wout, Chang, Sanfey, 2010).

La disregulación emocional se entiende como las dificultades para tener claridad respecto a qué emociones son las que están siendo experimentadas; fallas en la aceptación o tolerancia de emociones negativas; alteración en el comportamiento socialmente apropiado; alteración de la atención y el reconocimiento de las respuestas emocionales; y fallas en la utilización de estrategias apropiadas para la regulación emocional (Gratz y Roemer, 2004; Marín, Robles, González, Andrade, 2012).

Particularmente interesa la relación existente entre la disregulación emocional y las emociones morales, cuya interacción cursa de manera natural, pues la presencia o no de disregulación emocional en los sujetos insertos en ámbitos educativos, habrá de repercutir sobre la gestación de las emociones morales, y es en función de cómo está construida la experiencia subjetiva de la regulación emocional, que se pueden valorar las reglas morales sociales y el razonamiento moral del sujeto. Desde el ámbito educativo, esto se traduce en que los estudiantes comprendan las reglas, pautas y normas morales, por ejemplo respetar el reglamento escolar, respetar y conducirse conforme a los códigos de ética y conducta, entre otros.

De igual forma, estas normas morales habrán de analizarse en plenaria dentro del aula (grupo), para lograr la auto-reflexión, y considerar las consecuencias. Es decir, ¿qué pasaría si dichas normas no existieran? La intención, evidentemente, es llevar al estudiante no solo a conocer la importancia de estas reglas, sino también a la necesidad de respetarlas y buscar que los demás también las respeten. Para estos casos, se pueden realizar dinámicas grupales, aplicando estrategias tales como debates, foros de diálogo y deliberación, entre otros.

\section{Aportaciones desde la Neuropsicología al Ámbito Educativo}

Desde la multi e interdisciplinariedad, se rescatan contribuciones que la neuropsicología ha hecho a la educación, como las repercusiones del miedo y el estrés en el proceso de enseñanza-aprendizaje, a sabiendas de que, ante condiciones que se viven como amenazantes, es difícil prestar atención a otros estímulos, salvo a aquello que preocupa o atemoriza; o que el estrés crónico -sin estrategias compensatorias- afecta al almacenamiento de información en la memoria a largo plazo (Blanco, 2014); o la comprensión de que el aprendizaje se logra de manera más eficiente cuando el estado afectivo es óptimo, positivo, y permite alinearse con motivaciones personales.

Las aportaciones anteriores son ya conocidas y han permitido ir integrando poco a poco algunos programas -aunque escasos- para todos los interlocutores del ámbito educativo. En el caso de la formación en alumnos de nivel medio superior se encuentra el programa CONSTRUYE-T, de reciente implementación. En el caso de la formación continua en docentes, se cuentan con algunos programas de formación en emociones e interacciones socioafectivas.

Aunado a estas aportaciones, la neuropsicología también ha contribuido en el ámbito educativo a exponer por un lado la relevancia del desarrollo del aprendizaje social y la educación emocional, y por otro lado a identificar que para lograr éstos aprendizajes, se requiere volcar la mirada a procesos cognosocioafectivos que habían permanecido invisibles hasta ahora, como el procesamiento emocional.

Bajo la pertinencia del aprendizaje social, la educación emocional y el procesamiento emocional, se encuentra una nueva comprensión de los procesos cognosocioafectivos. Concebir al procesamiento 
REVISTA DE LA ESCUELA DE CIENCIAS DE LA EDUCACIÓN, AÑo 15, NRO. 14, VOL. 1, ENERO A JUNIO DE 2019. PÁGINAS 45-52. ISSN 1851-6297 (DESDE DICIEMBRE DE 2006 A DICIEMBRE DE 2017). ISSN 2362-3349 (EN LINEA). DISREGULACIÓN EMOCIONAL Y EMOCIONES MORALES EN EDUCACIÓN: APORTACIONES DESDE LA NEUROPSICOLOGÍA. CLAUDIA GODINEZ · DULCE MARÍA CAROLINA FLORES OLVERA.

emocional como proceso cognitivo, -según lo enuncia la neuropsicología-, permite amalgamar e integrar los aspectos cognitivos y socioafectivos para explicar la condición humana, y trascender la dicotomía existente entre éstos para empezar a hablar de procesos cognosocioafectivos.

Desde esta mirada, se recupera que las habilidades y destrezas sociales, emocionales y cognitivas características del hombre son posibles gracias a la actividad organizada y armónica de circuitos neuronales que forman ensambles o engranajes entre sí. A esta circuitería o red neuronal se le ha denominado "cerebro social" (Adolphs, 1999).

Es pues pertinente, para poder entender el rol de las emociones, rastrear la arquitectura funcional para el control cognitivo de la emoción, su correlato en las vías neurales y circuitos del cerebro (corticales y subcorticales), clarificando su relación anatomo-clínica (Bechara, Damasio, Damasio y Anderson, 1994; Hamman, Eli, Grafton, Kilts, 1999; Anderson y Phelps, 2000; Mora, 2000; Tucker, Derryberry, Luu, 2000; Gray y McNaughton, 2003; Greene, Nystrom, Engell, Darley, Cohen, 2004; Ochsner y Gross, 2005; Barret, Mesquira, Ochsner, Gross, 2007; Silva, 2008; Prehn, Wartenburger, Mériau, Scheibe, Goodenough, Villringer, van der Meer, Heekeren, 2008; Cáceda, James, Ely, Snarey, Kilts, 2011; Young y Dungan, 2010; Fumagalli y Priori, 2012).

Existen varios modelos neuropsicológicos y teorías que intentan explicar los mecanismos subyacentes a ciertas manifestaciones comportamentales, sin embargo, se rescatan aquéllos que resultan pertinentes por la aproximación que hacen hacia el sistema funcional del procesamiento emocional y particularmente la regulación emocional, como son: el Modelo del Marcador Somático, el Modelo Bioinformacional y la Hipótesis del Doble Proceso.

La tesis del modelo bioinformacional surge de la teoría de la emoción de Lang, y defiende que las emociones predisponen para la acción y que la respuesta emocional cumple principalmente una función social y otra motivacional (Bradley y Lang, 2000; Lang, 1985; citado en Chicharro, García, Sanjuán, 2011; Aguilar de Arcos, Montañez, Gómez, Arráez, Pérez, 2011).

La tesis del modelo del marcador somático expone que la toma de decisiones depende en muchos aspectos importantes de sustratos neurales que regulan la homeostasis, sin embargo, el ambiente influye en poner castigo o preferencia a nuestras elecciones, por lo que tales marcadores derivan de dispositivos neurales que se desarrollan en el proceso de educación y la adaptación social; esto es, no son innatos.

Por su parte, el Modelo de Greene, denominado Hipótesis del Doble Proceso, busca dar cuenta de los procesos emocionales y cognitivos involucrados en la toma de decisiones y la conducta moral. La pertinencia de este modelo radica en situar con igual importancia la participación, tanto de la emoción, como del razonamiento moral al momento de tomar una decisión (Greene, 2009), ya que varios estudios tanto conductuales (Greene, Haidt, 2002; Van't Wout, Chang, Sanfey, 2010; Pastötter, Gleixner, Neuhauser, Bäuml, 2012; Bedregal, León, Shand, Mosso, 2013) como de neuroimagen (Greene, Sommerville, Nystrom, Darley, Cohen, 2001; Bartels y Pizarro, 2011), muestran el papel central de la disregulación emocional sobre la construcción y expresión de las emociones morales, por ejemplo, ante la toma de decisiones de dilemas morales.

Al respecto no se pretende caer ni en determinismos ni biologicismos. Si bien se sabe que las emociones tienen una base biológica, también se sabe que el proceso cognosocioafectivo no puede ser reducido a la parte orgánica, sino que se construye en una interacción social y psicológica inmersas en una cultura. Sin ir más lejos, "se ha mostrado que la experiencia, adquirida en la ontogenia temprana, modifica esencialmente el desarrollo bioquímico, morfológico y fisiológico del cerebro y la conducta" (Venguer y Ibatullina, 2010, s/p).

Una vez conocidas las tesis anteriores, que revelan cómo la afectación del mecanismo emocional provoca malas decisiones, podrá comprenderse mejor la relación entre la disregulación emocional y las emociones morales. Así mismo, y como se ha mencionado, la aproximación neuropsicológica ha enriquecido al escenario educativo, llevándolo al diálogo interdisciplinar y a eliminar escisiones entre procesos cognitivos y socioafectivos, lo cual lleva a cuestionarnos: ¿Hacia qué convergencias y nuevas aproximaciones nos invita dicho diálogo?

\section{Relación entre la disregulación emocional y las emociones morales: la aproximación neuropsicológica}

Para responder a lo anterior, en primera instancia, se reconocen nuevos procesos como el procesamiento emocional, bajo un amplio panorama que permite comprender que los procesos cognosocioafectivos tienen un fundamento cerebral, y deben ser integrados en la formación del estudiante. A manera de ejemplo, pocas veces se le pregunta al estudiante qué significado tiene para él este conocimiento, que valoración le da a su desempeño, o cómo podría mejorarlo, entre otros. Estos cuestionamientos deberían ser también indicadores fundamentales del proceso educativo, aunado a que motivan a respetar y posicionar al estudiante como centro del proceso educativo, y acercan al profesor al desarrollo verdaderamente integral del estudiante.

En segundo lugar se comprende que el sentido central de los procesos cognioscioafectivos no es la culminación del desarrollo social, sino la condición previa para ello. Si el ser humano no sintiera esa conexión inicial desde el nacimiento gracias a la reciprocidad y conexión percibida con el otro, las interacciones no podrían tomar su forma tan distintivamente humana (Meltzoff, 2013). Esto es, podemos provocar y promover esos procesos de enseñanza-aprendizaje cognosocioafectivos porque estamos determinados -aunque no terminados- para entablar relaciones emocionales. La naturaleza humana es 
REVISTA DE LA ESCUELA DE CIENCIAS DE LA EDUCACIÓN, AÑo 15, NRO. 14, VOL. 1, ENERO A JUNIO DE 2019. PÁGINAS 45-52. ISSN 1851-6297 (DESDE DICIEMBRE DE 2006 A DICIEMBRE DE 2017). ISSN 2362-3349 (EN LINEA). DISREGULACIÓN EMOCIONAL Y EMOCIONES MORALES EN EDUCACIÓN: APORTACIONES DESDE LA NEUROPSICOLOGÍA. CLAUDIA GODINEZ · DULCE MARÍA CAROLINA FLORES OLVERA.

en sí misma bio-psico-social-espiritual, y permite que seamos capaces de aprender a relacionarnos, ser empáticos, o a expresar asertivamente las emociones.

Y como tercer punto, entender que cuando no se tienen, o no se han enseñado las estrategias correctas para la expresión emocional, entra en escena el fenómeno de disregulación emocional, mismo que impacta sobre la conducta moral esperada.

La disregulación emocional afecta a las emociones morales, porque dichas emociones morales invitan siempre a tener una postura frente a algún fenómeno, y esta postura invariablemente irá cargada de juicios de valor positivos o negativos, acordes o no, a las normas sociales. Así pues, la postura que se decida tomar, será un proceso guiado por señales emocionales; sin embargo, si ese mecanismo emocional se ve afectado, entonces provocará decisiones erróneas, basadas en el reforzamiento inmediato (miopía hacia el futuro), así como persistencia en el error, debido a la dificultad para incorporar aprendizajes afectivos a decisiones posteriores (Damasio, Tranel, Damasio, 1991; Damasio, 1994; Bechara y Damasio, 2005; Verdejo, Pérez, Bechara, 2006).

Para traducir lo previamente dicho al ámbito educativo, pensemos en un joven que presenta disregulación emocional, por lo que sus emociones morales y sus decisiones estarán basadas principalmente en el reforzamiento inmediato. Esto habrá de colaborar en fallas importantes para apegarse a las normas socialmente establecidas, por lo que el joven podrá inclinarse hacia la toma de decisiones impulsivas como la deserción escolar, o colocarse en situaciones de riesgo como el acercamiento temprano a las drogas, o conductas deshonestas como el plagio.

Al respecto, es muy importante reconocer que la disregulación emocional no es el único factor involucrado en fenómenos como la deserción escolar, las drogas o el plagio; sin embargo, es un factor de riesgo que se suma, y que por ende debería ser plenamente estudiado, pues lo que sí es un hecho, es que la disregulación emocional provoca tanto una disfunción en la emoción como en la conducta moral social esperada. Esto es así porque la disregulación emocional provoca

dificultades para identificar experiencias emocionales específicas, modular la activación fisiológica asociada, tolerar el malestar y confiar en las respuestas emocionales como interpretaciones válidas de experiencia vitales, por ende, la disregulación emocional condiciona a la autoinvalidación y desorienta respecto a la manera de actuar y sentir en general (Linehan,1993; citado en Marín, Robles, González, Andrade, 2012, s/p).

Dicho de otro modo, la disregulación emocional no propicia un autoaprendizaje en el que se pueda confiar, por el contrario, desencadena incertidumbre respecto al propio sentir, e impacta de forma negativa a todo tipo de emoción, con hincapié sobre las emociones morales por su representatividad educativa y social.

Por poner ejemplos altamente relevantes en el proceso educativo, en las emociones morales de condena, una buena regulación emocional habrá de conducir a conductas pro-sociales; por el contrario, la movilización a la acción desde la disregulaición emocional puede generar conductas egoístas o antisociales. Otros efectos de la disregulación emocional, respecto a las emociones morales de autoconciencia (vergüenza, pudor y culpa), incluye la reducción de la presencia social y en casos extremos el suicidio; en el caso de las emociones morales relativas al sufrimiento ajeno, la disregulación emocional impide la compasión; y respecto a las emociones morales de admiración, la disregulación emocional impide forjar relaciones y habilidades sociales cohesivas (Haidt, 2003; citado en Mercadillo Díaz, Barrios, 2007).

La relación entre las emociones morales y la disregulación emocional se vincula con procesos de enseñanza-aprendizaje de corte cognosocioafectivo, porque la disregulación emocional provoca alteraciones de los mecanismos reguladores y de control inhibitorio de la conducta, produce fallas en la anticipación y el establecimiento de metas, el diseño de planes, la inhibición de respuestas inapropiadas, la adecuada selección de conductas y su organización en el espacio y en el tiempo, la flexibilidad cognitiva en la monitorización de estrategias, la supervisión de las conductas en función de estados motivacionales y afectivos, y la toma de decisiones (Verdejo et al, 2004; citado en García, García y Secades, 2011).

Al respecto, la literatura concede un papel central a la disregulación emocional sobre las emociones morales, de las que se desprende una biunívoca relación con los juicios y dilemas morales (Greene, Sommerville, Nystrom, Darley, Cohen, 2001; Bartels y Pizarro, 2011; Pastötter, Gleixner, Neuhauser, Bäuml, 2013). El impacto que genera la disregulación emocional sobre las emociones morales, produce un sesgo atencional al momento del aprovechamiento de oportunidades y enfrentamiento de desafíos de manera efectiva en el entorno. Por ende, se enfatiza en prestar atención en el ámbito educativo a la disregulación emocional y su relación con las emociones morales, ya que su presencia conduce al aislamiento, llevando al individuo a emitir conductas instintivas, desinhibición y juicios morales inapropiados, deterioro de la autoconciencia y decisiones impulsivas (Moeller et al, 2012; Murphy et al, 2012).

El estudio en la disregulación emocional sobre las emociones morales de los interlocutores del proceso de enseñanza-aprendizaje nos acerca a una mayor comprensión de ciertas conductas desadaptativas y antisociales. Si bien la disregulación emocional no es el único elemento causal de tales conductas desadaptativas, sí es un elemento clave que contribuye en gran medida a que se presenten. Lo anterior se traduce en prácticas lamentablemente comunes en el escenario educativo como el plagio, juicio empobrecido, respuestas impulsivas, falta de empatía, quebrantamiento constante de reglas y 
REVISTA DE LA ESCUELA DE CIENCIAS DE LA EDUCACIÓN, AÑo 15, NRO. 14, VOL. 1, ENERO A JUNIO DE 2019. PÁGINAS 45-52. ISSN 1851-6297 (DESDE DICIEMBRE DE 2006 A DICIEMBRE DE 2017). ISSN 2362-3349 (EN LINEA). DISREGULACIÓN EMOCIONAL Y EMOCIONES MORALES EN EDUCACIÓN: APORTACIONES DESDE LA NEUROPSICOLOGÍA. CLAUDIA GODINEZ · DULCE MARÍA CAROLINA FLORES OLVERA.

obligaciones sociales, e inclusive autolesiones no suicidas o el uso y abuso de sustancias, por poner algunos ejemplos.

\section{Conclusiones}

Las neurociencias y particularmente la neuropsicología, han aportado conocimientos fundamentales sobre el funcionamiento del cerebro, la valoración e identificación de las alteraciones cognitivas que impactan en trastornos del aprendizaje, etc. Pero sobre todo en las bases neurales del proceso de enseñanza-aprendizaje, y su relación con los procesos cognosocioafectivos y morales.

Tradicionalmente se ha hecho una división entre la razón y la emoción; sin embargo, para la completa comprensión de la aportación del estudio de las emociones dentro del ámbito educativo, se les debe entender como elementos de un todo: de procesos cognosocioafectivos, cuyo estudio requiere de sistematización y rigurosidad. Esto permitirá entender que el estudio en emociones no tiene sólo como objetivo buscar que los sujetos "se sientan bien", sino brindar estrategias particulares para su claro reconocimiento y expresión.

Por lo anterior, se hace hincapié en incluir cada vez más en la agenda educativa tópicos como las emociones y, en este caso, la relación entre disregulación emocional y emociones morales, pues los hallazgos permitirán generar una concepción más detallada y específica en el escenario educativo respecto a la construcción e inmersión en las normas sociales, y el apego que se tenga hacia conductas morales y pro-sociales.

Así pues, la vinculación entre las emociones morales y la disregulación emocional resulta un factor de estudio muy relevante en el fenómeno de la educación. Por un lado, porque la presencia de disregulación emocional en las emociones morales irrumpe con los códigos éticos y morales, relacionándose con sesgos en el sistema de creencias y valores del alumnado y de los docentes; también se asocia con la transgresión a la norma, impactando la percepción subjetiva del quebrantamiento de normas sociales explícitas e implícitas, así como de estereotipos inherentes en los códigos, actitudes y creencias individuales.

Por otro lado, conocer más sobre la relación entre la disregulación emocional y las emociones morales no es anodina, porque los modelos de prevención sugieren de manera global, reducir las fuentes de estrés del entorno y desarrollar las capacidades del sujeto para potenciar una mejor gestión de la toma de decisiones; desarrollar una mayor resiliencia y facilitar estrategias de afrontamiento que permitan reducir el malestar y el distrés asociado a los eventos cotidianos. La relación entre disregulación emocional y su presencia en emociones morales, podría contribuir para brindar una explicación y un panorama más amplio sobre la constante falta de respeto a las reglas sociales o morales, de los códigos éticos, de conducta, entre otros.

Finalmente, debe entenderse que los mecanismos neurales que subyacen a la emoción, también participan en todos los demás procesos cognitivos. El aporte estriba en reconocer el valor y la necesidad de incluir a las emociones como parte de los procesos cognosocioafectivos, y como elemento relevante dentro del ámbito educativo, reconociendo su papel en el desarrollo integral de los estudiantes, y contribuir en la generación de una línea de investigación que busca articular y profundizar el tratamiento epistemológico del proceso educativo.

\section{Referencias bibliográficas}

- $\quad$ Aguilar de Arcos, F; Montañez, M; Gómez, E; Arráez, F. y Pérez, M. (2011). "Influencia del contenido emocional en la percepción de estímulos visuales en sujetos drogodependientes". Trastornos Adictivos, 13 (2), pp. 57-63.

- Anderson, A.K. y Phelps, E.A. (2000). "Expression without recognition: Contributions of the human amygdala to emotional communication". Psychology Science, 11, pp. 30-38.

- $\quad$ Barret, L.F; Mesquita, B; Ochsner, K.N. y Gross, J.J. (2007). "The experience of emotion". Annu Rev Psychol, 58, pp. 373-403.

- Bartels, D y Pizarro, D. (2011). "The mismeasure of morals: Antisocial personality traits predict utilitarian responses to moral dilemas". Cognition. doi:10.1016/j.cognition.2011.05.010

- Bechara, A; Damasio, A; Damasio, H. y Anderson, S. (1994). "Insensitivity to future consequences following damage to human prefrontal cortex". Cognition, 50: pp. 7-15. doi: 10.1016/0010-0277(94)90018-3

- Bechara, A. y Damasio, A. (2005). "The somatic marker hyphotesis: A neural theory of economic decision". Games and Economic Behavior, 52, pp. 336-372.

- Blanco, I. (2014). Mindware Neuropsicología aplicada a la educación. Editorial Morsan Internacional S.A. de C.V, México.

- $\quad$ Bradley, M.M. y Lang, P.J. (2000). "Memotion: behavior, feeling and physiology". En Lane, R; Nadel, L. (Eds.), Cognitive Neuroscience of Emotion, pp. 242-276. Oxford University Press, Nueva York.

- Bradley, M.M. y Lang, P.J. (2006). "Motivation and emotion”. En Cacioppo, J.T., Tassinary, L.G. y Berntson, G. (Eds.), Handbook of Psychophysiology, pp.581-607. Cambridge University Press, New York.

- $\quad$ Cáceda, R; James, A; Ely, T; Snarey, J. y Kilts, C. (2011). "Mode of Effective Connectivity within a Putative Neural Network Differentiates Moral Cognitions Related to Care and Justice Ethics". PLos ONE, 6 (2): e14730, 1-12. doi:10.1371/journal.pone.0014730

- Chicharro, J; Pérez García, A. y Sanjuán, P. (2011). "Respuesta emocional en adictos a sustancias en 
REVISTA DE LA ESCUELA DE CIENCIAS DE LA EDUCACIÓN, AÑo 15, NRO. 14, VOL. 1, ENERO A JUNIO DE 2019. PÁGINAS 45-52. ISSN 1851-6297 (DESDE DICIEMBRE DE 2006 A DICIEMBRE DE 2017). ISSN 2362-3349 (EN LINEA). DISREGULACIÓN EMOCIONAL Y EMOCIONES MORALES EN EDUCACIÓN: APORTACIONES DESDE LA NEUROPSICOLOGÍA. CLAUDIA GODÍNEZ · DULCE MARÍA CAROLINA FLORES OLVERA.

tratamiento ambulatorio". Adicciones, 24 (1), pp. 59-68.

- Damasio, A. (1994). El error de Descartes: Emoción y razonamiento en el cerebro humano. Crítica, Barcelona.

- Damasio, A. (2005). En busca de Spinoza: Neurobiología de la emoción y de los sentimientos. Crítica, Barcelona.

- Damasio, A; Tranel, D. y Damasio, H. (1991). "Somatic Markers and the Guidance of Behavior: Theory and Preliminary Testing”. En Levin, H.S; Eisenberg, H.M; Benton, A.L. (Eds.), Frontal Lobe Fuction and Dysfuction, pp.217-229. Oxford University Press, USA.

- $\quad$ Fernández-Abascal, E; Jiménez Sánchez, M.P. y Martín Díaz, M.D. (2003). Emoción y motivación. Ramón Areces, Madrid.

- Fumagalli, M. y Priori, A. (2012). "Functional and clinical neuroanatomy of morality". Brain, 1-16. doi: 10.1093/brain/awr334

- García, G; García, O. y Secades, R. (2011). “Neuropsicología y Adicción a Drogas”. Papeles del Psicólogo, 32 (2), 159-165.

- Gratz, K. y Roemer, L. (2004). "Multidimensional Assessment of Emotion Regulation and Dysregulation: Development, Factor Structure, and Initial Validation of the Difficulties in Emotion Regulation Scale". Journal of Psychopathology and Behavioral Assessment, 26 (1), pp. 41-54.

- $\quad$ Gray, J.A. y McNaughton, N. (2003). The Neuropsychology of Anxiety: An Enquiry into the Functions of the Septo-Hippocampal System. Oxford University Press, Great Britain.

- $\quad$ Greene, J; Nystrom, L; Engell, A; Darley, J y Cohen, J. (2004). "The Neural Bases of Cognitive Conflict and Control in Moral Judgment". Neuron, 44, pp. 389-400.

- $\quad$ Greene, JD; Sommerville, R; Nystrom, L; Darley, J. y Cohen, J.(2001). "An fMRI investigation of emotional engagement in moral judgment". Science, 14 (293), 2105-8.

- Hamman, S.B; Eli, T.D.; Grafton, S.T. y Kilts, C.D. (1999). "Amygdala activity related to enhanced memory for pleasant and aversive stimuli”. Nature Neuroscience, 2, pp. 289-303.

- Hernández Galván, A. (2014). Evaluación de la cognición social en adultos mayores de la Ciudad de México. (Tesis de Doctorado no publicada). Universidad Nacional Autónoma de México. México, D.F.

- LeDoux, J. (2012). "Rethinking the emotional brain". Neuron, 73 (4), 653-676. doi 10.1016/j.neuron.2012.02.004.

- Marín Tejeda, M; Robles García, R; González-Forteza, C. y Andrade Palos, P. (2012). "Propiedades psicométricas de la escala "Dificultades en la Regulación Emocional" en español (DERS-E) para adolescentes mexicanos". Salud Mental, 35 (6), pp. 521-526.

- Martínez, S.F. (2008). "La emoción". En Palmero F y Martínez S.F. (2008). Motivación y emoción. McGrawhill, España.

- Meltzoff, A.N. (2013). "Origins of social cognition: Bidirectional self-other mapping and the "Like-Me" hypothesis" In M. Banaji \& S. Gelman (Eds.), Navigating the social world: What infants, children, and other species can teach us, pp. 139-144. Oxford University Press, New York.

- Mercadillo, R; Díaz, J. y Barrios, F. (2007). "Neurobiología de las emociones morales". Salud Mental, 30 (3), pp. 1-11.

- Moeller, S; Hajcak, G; Parvaz, M; Duning, J; Volkow, N. y Goldstein, R. (2012). "Psychophysiological prediction of choice: relevance to insight and drug addiction". Brain, 135, 3481-1494. doi: 10.1093/brain/aws252.

- $\quad$ Mora, F. (2000). El cerebro sintiente. Ariel Neurociencia, España.

- Murphy, A; Taylor, E. y Elliott, R. (2012). "The detrimental effects of emotional process dysregulation on decisión-making in substance dependence". Frontiers in Integrative Neuroscience, 6, 1-24. doi: 10.3389/fnint.2012.00101

- $\quad$ Ochsner, K.N. y Gross, J.J. (2005). "The cognitive control of emotion”. Trends Cogn Sci, 9, pp. 242-249.

- $\quad$ Pastötter, B; Gleixner, S; Neuhauser, T. y Bäuml, KH. (2013). "To push or not to push? Affective influences on moral judgment depend on decision frame". Cognition, 126 (3), 373-7. doi: 10.1016/j.cognition.2012.11.003.

- $\quad$ Prehn, K; Wartenburger, I; Mériau, K; Scheibe, C; Goodenough, O; Villringer, A; van der Meer, E. y Heekeren, H. (2008). "Individual differences in moral judgment competence influence neural correlates of socio-normative judgments". SCAN, 3, 33-46. doi:10.1093/scan/nsm037

- Silva, J. (2008). "Neuroanatomía Funcional de las Emociones". En Slachevsky, A; Manes, F; Labos, E; Fuentes, P. (Eds). Tratado de Neuropsicología y Neuropsiquiatría Clínica, pp 377-384. Akadia, Chile.

- Tucker, D, Derryberry, D, y Luu, P. (2000) "Anatomy and Physiology of Human Emotion: Vertical Integration of Brain Stem, Limbic, and Cortical Systems". En Borod, J.C. (Ed.), The Neuropsychology of Emotion, pp.56-79. Oxford University Press, USA.

- Van't Wout, M; Chang, L. y Sanfey, A. (2010). "The influence of emotion regulation on social interactive decision-making". Emotion, 10 (6), pp. 815-821. doi: 10.1037/a0020069

- Venger, L. y lbatullina, A. (2010). "La correlación entre la enseñanza, el desarrollo psicológico y las particularidades funcionales de la maduración cerebral" En Solovieva, Y: Quintanar, L. (Comps.) Antología del desarrollo psicológico del niño en edad preescolar, pp.40-45. Trillas, España.

- Verdejo, A. y Bechara, A. (2009). "A somatic marker theory of addiction”. Neuropharmacology, 56, pp. 4862.

- Verdejo, A; Pérez García, M. y Bechara, A. (2006). "Emotion, decision-making and substance dependence: A somatic-marker model of addiction”. Current Neuropharmacology, 4(1): pp. 17-31.

- Young, L. y Dungan, J. (2010). "Where in the brain is morality? Everywhere and maybe Nowhere". Social Neuroscience. doi: 1080/17470919.201 